\title{
Detection of Lung Cancer Lesions Using 3D Convolutional Neural Networks and Segmentation for Accurate Detection
}

\author{
Rajani Kumari ${ }^{1}$, C. Thanuja ${ }^{2}$, K. Sai Thanvi ${ }^{3}$, K. Lakshmi ${ }^{4}$, U. Lavanya ${ }^{5}$ \\ ${ }^{1}$ Assistant Professor, Department of Electronics and Communication Engineering, Sanskrithi School of \\ Engineering Puttaparthi, Andhra Pradesh, India \\ 2,3,4,5B. Tech Students, Department of Electronics and Communication Engineering, Sanskrithi School of \\ Engineering, Puttaparthi, Andhra Pradesh, India
}

\section{Article Info \\ Volume 8, Issue 4 \\ Page Number : 435-439}

\section{Publication Issue}

July-August-2021

\section{Article History}

Accepted : 20 July 2021

Published : 27 July 2021

\section{ABSTRACT}

Lung cancer is a leading cause of death worldwide; it refers to the uncontrolled growth of abnormal cells in the lung. A computed tomography (CT) scan of the thorax is the most sensitive method for detecting cancerous lung nodules. A lung nodule is a round lesion which can be either non-cancerous or cancerous. In the CT, the lung cancer is observed as round white shadow nodules. In existing method, the candidate ROIs shape features are calculated, and some blood vessels are get rid of using rule-based according to shape features; secondly, the remainder candidates gray and texture features are calculated; finally, the shape, gray and texture features are taken as the inputs of the SVM (Support Vector Machine) classifier to classify the candidates. Experimental results show that the rule-based approach has no omission, but the misclassification probability is too large; Hence, in the proposed method the nodules were characterized by the computation of the texture features obtained from the gray level co-occurrence matrix (GLCM) in the wavelet domain and were classified using a SVM with radial basis function in order to classify CT images into two categories: with cancerous lung nodules and without lung nodules. The stages of the proposed methodology to design the CADx system are: 1) Extraction of the region of interest, 2) Wavelet transform, 3) Feature extraction, 4) Attribute and sub-band selection and 5) Classification. The same classification is implemented for the convolution neural networks. The final comparison is done between these two networks based on the accuracy.

Keywords : Lung Cancer, Convolutional Neural Network, Tensorflow, CT Scan

\section{INTRODUCTION}

Lung cancer is one of the most-fatal diseases all over the world today. About 1.8 million people have been suffering from lung cancer in the whole world [1]. In the United States, only $17 \%$ of people are diagnosed with lung cancer and they sur-vived for five years after the diagnosis. But the survival rate is lower in 
develop-ing countries [2]. The growth of uncontrolled cells can spread beyond the lung by the process of metastasis into nearby tissue or other parts of the body [3]. The cancer is localized to the lungs at the first two stages and is spread out to different organs in the latter stages. The diagnostic methods are CT scans (Computerized Tomography), chest radiography (Xray), MRI scan (Magnetic Resonance Im-aging) and biopsies etc. However, it is difficult to detect lung cancer in the early stage. In this research, we have collected CT scan images of 1500 patients. But we have worked on the CT images of 100 patients where each of them contains more than 120 DICOM 3D images. Among these, 80 patients' images are used here for training purposes and 20 patients' images are used for testing purpose.Our system is robust as well as effective for the early detection of lung cancer.

This research contributes to the following:

1) A literature survey is performed on the existing state-of-the-art techniques

for the detection of lung cancer.

2) A comprehensive study is performed with standard dataset using deep convolutional neural network architectures for lung cancer detection in the early stage.

\section{LITERATURE REVIEW}

S. Logesh kumar1, M. Swathy, S. Sathish, J. Sivaraman and M. Rajasekar, "Identification of Lung Cancer Cell using Watershed Segmentation on CT Images", Indian Journal of Science and Technology, Vol9(1),DOI:10.17485/ijst/2016/v9i1/85765, January 2016.

- In this experiment, various Computed Tomography (CT) images of the lung were used as input images with pixel size of $512 \times 512$ and obtained the output image in JPEG format.

- Compared to X-ray CT images are perceptive characteristics of identifying lung tumor size and lymph nodes.
- Thresholding selects a threshold value $\mathrm{T}$ and it assigns two levels to the image that is above value and below value for original threshold value.

- FFT, Auto enhancement and Gabor filtering are the three methods used as image enhancement techniques.

- Based on this study it is more evident that watershed segmentation technique is good for evaluation of lung cancer cell region.

\section{Methodology}

\section{1) Convolutional layer:}

This layer is where images are translated into featuremap data by convolu-tional kernels or filters. In a 3D $\mathrm{CNN}$, the kernels move through three dimen-sions of data (height, length, and depth) and produce 3D maps. A $3 \mathrm{D} C N N$ is necessary for analyzing data where temporal or volumetric context is important.

\section{2) Pooling layer:}

Pooling, or down-sampling, is done on the convolutional output. During pooling, a filter moves across the convolutional output to take either the average or the weighted average or the maximum value. The goal of the pooling layer is to progressively reduce the spatial size of the matrix to reduce the number of pa-rameters and to control over fitting.

\section{3) Fully-connected layer:}

The fundamental goal of a fully connected layer is to take the results of the convolution and pooling processes and use them to classify the image into a label. In this layer, a softmax function is used to get probabilities as it pushes the values between 0 and 1 . Batch normalization is used to improve the training speed and to reduce overfitting.

\subsection{Proposed Methodology:}


The proposed method consists of the convolution neural network which is a deep learning technique used for classification processes. The convolution neural network and its layers is explained below:

This architecture consists of 7 layers. The input images are fed to the network taking 50 images as a batch at a time. All images are resized to have $32 \times 32$ dimensions and all these images are true color or RGB images. And the layers of DCCN are three convolutional layers, three pooling layers and a fully connected layer.

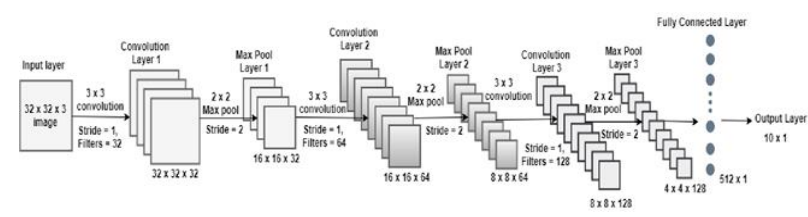

Fig.3.1:Block Diagram of Proposed Method

\subsection{Datasets:}

We used LUNA16 (Lung Nodule Analysis) datasets (CT scans with labeled nodules). The LUNA 16 dataset has the location of the nodules in each CT scan. Thus, it will be useful for training the classifier. The inputs are the image files that are in "DICOM" format. The format and configuration of the images are different since the images are captured at different time and from different types of the camera. Actually, the images are of size $(\mathrm{z} \times 512 \times 512)$, where $\mathrm{z}$ is the number of slices in the CT scan and varies depending on the resolution of the scanner [13]. Such large images cannot be fed directly into convolutional neural network architecture because of the limit on the computation power. At first, we converted all the images into similar size and format. To reduce the size of the input data, we have segmented the image. Thus, we have to find the regions that are more probable to have cancer. We have reduced our search space by first segmenting the lungs and then removing the low intensity regions. We have used the pixel as input to the neural network. The total size of the input data was 15 GB. A small subset of data of size around 2 GB has used for various testing purposes.

\section{Experimental Results}
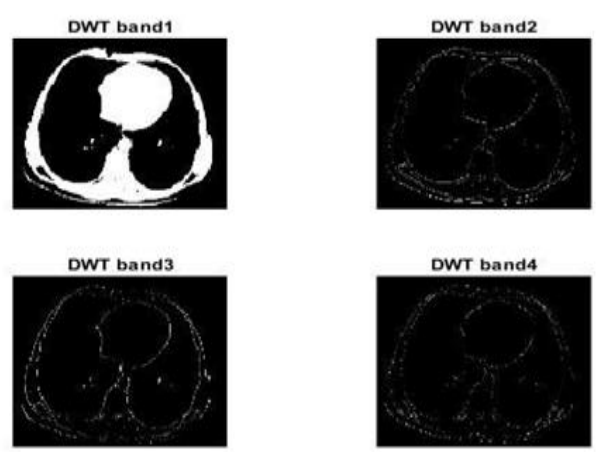

Fig.4.1: Input and output images of normal CT image
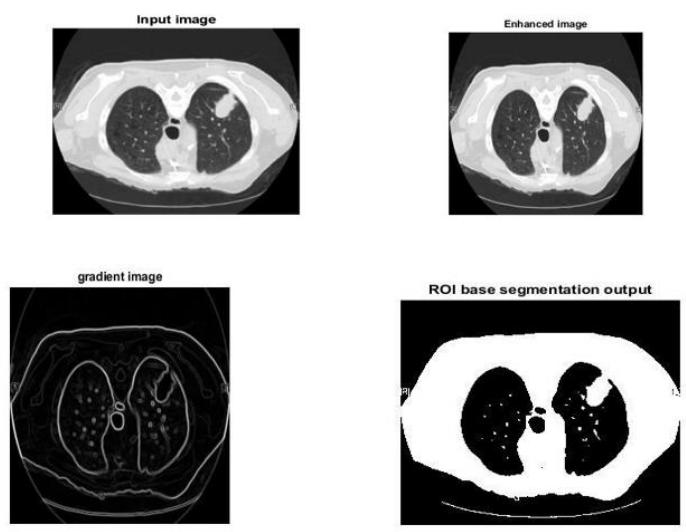

Fig.4.2:shows Tumor present around corners of lung
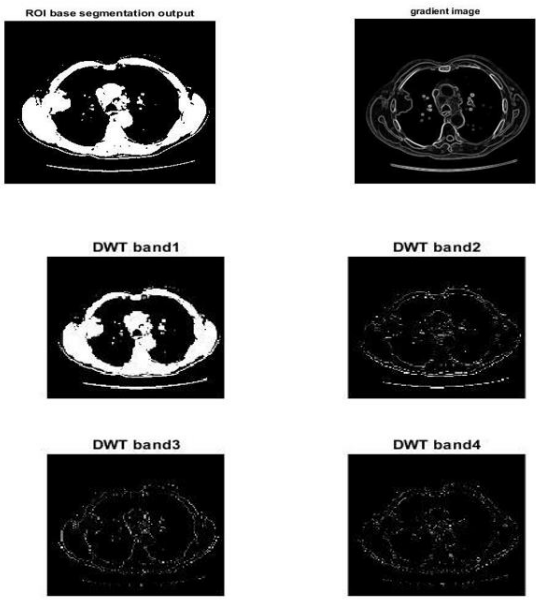

Fig.4.3:Input (CT image) and output images for lung where tumor is present in bronchioles 


\section{Future Work}

In this research, we used a vanilla 3D CNN classifier to determine whether a CT image of lung is cancerous or non-cancerous. Before using the $3 \mathrm{D} \mathrm{CNN}$, we preprocessed the CT image through a thresholding technique. We have performed a thorough experiment using LUNA 16 dataset. Our obtained detection accuracy is $80 \%$, which is better than existing methods. In future, we will perform the experiments on a large amount of data and apply more features such as nodule size, texture and position for further improvement. We will also try to apply the state-of-the-art deep CNN methods for higher accuracy and use our method on other types of cancer detection.

\section{Conclusion}

The 3D structure of nodules is used to extract features by 3D CNN to predict lung cancer. By using CNNs, the tedious task of manually extracting features can be eliminated. The obtained precision and recall obtained for the model are decent considering that less labelled data than most state-of-the-art CAD systems are used.

\section{VII.REFERENCES}

[1]. Diciotti S, Lombardo S, Falchini M, Picozzi G, Mascalchi M. "Automated segmentation refinement of small lung nodules in CT scans by local shape analysis". IEEE T Bio-Med Eng.vol.58,12,pp:3418-

28.doi:10.1109/TBME.2011.2167621. Dec. 2011.

[2]. Lee M, Boroczky L, Sungur K, Cann A, Borczuk A, Kawut S, et al." Computer-aided diagnosis of pulmonary nodules using a two-step approach for feature selection and classifier ensemble construction”. Artif Intell Med. Vol.50,1, pp:4353. doi: 10.1016/j.artmed.2010.04.011. Sep.2010.

[3]. Van de Wouwer G, Scheunders P, Van Dyck D. "Statistical texture characterization from discrete wavelet representations". IEEE T Image Process. Vol.8,4, pp:592-8. doi: 10.1109/83.753747. 1999.

[4]. Singh R, Khare A. 'Fusion of multimodal medical images using daubechies complex wavelet transform: a multiresolution approach". Inform Fusion. 2014.

[5]. Armato S, McLennan G, Bidaut L, McNitt-Gray $\mathrm{M}$, Meyer C. "The lung image database consortium (LIDC) and image Database resource initiative (IDRI)": a completed reference database of lung nodules on CT scans. Medical Physics vol.38,2, pp:915-931. Jan.2011.

[6]. Jing $Z$, Bin L, Lianfang $T$. "Lung nodule classification combining rule-based and SVM." In: Edited by Li K, Proceedings of the IEEE Fifth International Conference on Bio-Inspired Computing: Theories and Applications: 23-26 September 2010; Changsha, China. Piscataway, NJ: IEEE Computer Society; 2010.

[7]. Zhu Y, Tan Y, Hua Y, Wang M, Zhang G, Zhang J. 'Feature selection and performance evaluation of support vector machine (svm)based classifiers for differentiating benign and malignant pulmonary nodules by computed tomography". Journal of Digital Imaging vol.23,1, pp:51-65, March 2009;

[8]. Chen H, Zhang J, Xu Y, Chen B, Zhang K. "Performance comparison of artificial neural network and logistic regression model for differentiating lung nodules on CT scans". Journal of Biomedical Science and Engineering, Vol.6 No.11, November 25, 2013.

[9]. Nidhi S. Nadkarni, Prof. Sangam Borkar, "Detection of Lung Cancer in CT Images using Image Processing", Third International Conference on Trends in Electronics and Informatics, IEEE, 2019. 
[10].Kumar SA, Ramesh J, Vanathi PT, Gunavathi $\mathrm{K}$."Robust and automated lung nodule diagnosis from CT images based on fuzzy systems". In: Edited by Manikandan V, Proceedings of the IEEE International Conference on Process Automation, Control and Computing: 20-22 July 2011; Coimbatore, India. Piscataway, NJ: IEEE Women in Engineering; 2011.

[11].Keshani M, Azimifar Z, Taheripour F, Boostani R. "Lung nodule segmentation and recognition using SVM classifier and active contour modeling": a complete intelligent system Comput Biol Med. Vol.4,4, pp:287-300. doi: 10.1016/j.compbiomed.2012.12.004.2013.

[12].S.Logesh kumar1, M. Swathy, S. Sathish, J. Sivaraman and M. Rajasekar, "Identification of Lung Cancer Cell using Watershed Segmentation on CT Images", Indian Journal of Science and Technology, Vol.9,1, DOI:10.17485/ijst/2016/v9i1/85765, January 2016.

[13].Janee Alam1, Sabrina Alam2, Alamgir Hossan3 "Multi-Stage Cancer Detection and Prediction Using Multi-class SVM classifier", International Journal of Computer Science and Information Technologies, Vol.4, issue 1, pp:39-45.2018.

[14].Kuruvilla J, Gunavathi K. "Lung cancer classification using neural networks for CT images". Comput Meth Prog Bio. Vol.113, issue 1, Jan.2014.

[15].Gigliarano C, Figini S, Muliere P. "Making classifier performance comparisons when ROC curves intersect". Comput Stat Data An. Vol. 77, pp:300-12.743:287-300. 2014.

\section{Cite this article as :}

Rajani Kumari, C. Thanuja, K. Sai Thanvi, K. Lakshmi, U. Lavanya, "Detection of Lung Cancer Lesions Using 3D Convolutional Neural Networks and Segmentation for Accurate Detection", International Journal of Scientific Research in Science and Technology (IJSRST), Online ISSN : 2395-602X, Print ISSN : 23956011, Volume 8 Issue 4, pp. 435-439, July-August 2021. Available at

doi : https://doi.org/10.32628/IJSRST218442

Journal URL : https://ijsrst.com/IJSRST218442 